\title{
Alive Animal Model for Epilepsy by Intradorsal Striatum Injection of Colchicine
}

\author{
Zahra Fakhroleslam ${ }^{1}$ Manizheh Karami ${ }^{1}$ Mehrdad Roghani $\quad$ Atiyeh Nejadebrahim ${ }^{1}$
}

${ }^{1}$ Department of Biology, Faculty of Basic Sciences, Shahed University, Tehran, Iran

2Department of Physiology, Faculty of Medicine, Shahed University, Tehran, Iran

Int J of Epilep 2021;7:8-14.

\begin{abstract}
Address for correspondence Manizheh Karami, PhD, Department of Biology, Faculty of Basic Sciences, Shahed University, Tehran, Iran (e-mail: karami@shahed.ac.ir).
\end{abstract}

\begin{abstract}
Keywords

- epilepsy

- colchicine

- dorsal striatal region

- alive

- rat

Background Epilepsy is a neural disorder with repeatable seizure attacks. In this article, we used the neurotoxin colchicine, which is derived from the plant Colchicum autumnale, to introduce a low cost but the more valuable alive animal model for epilepsy. Materials and Methods Wistar rats weighing 250 to $300 \mathrm{~g}$ after intraperitoneal injection of ketamine $(100 \mathrm{mg} / \mathrm{kg})$ and xylazine $(20 \mathrm{mg} / \mathrm{kg})$ were restrained in the stereotaxic apparatus; they were cannulated in the dorsal striatal area (AP: $0.5 \mathrm{~mm}$; $\mathrm{L}: 3 \mathrm{~mm}$; V: $3.6 \mathrm{~mm}$ ). One week later, an injection cannula attached to a $5-\mu$ Hamilton syringe by polyethylene tubing guided 0.05 to $25 \mu \mathrm{g} /$ rat colchicine in the recovered healthy rats once daily for 4 consecutive days. The control group solely received the saline solution. The behavioral signs of all animals were daily recorded. Finally, the brains of rats under deep euthanasia were collected in $10 \%$ formalin and examined histopathologically. The dorsal striatal regions were cut coronally into 3 to $4 \mu \mathrm{m}$-thick slices, and then stained with hematoxylin-eosin. They were eventually examined under the light microscope to verify the injection placement or possibility of lesions. All data were analyzed by analysis of variance under $\alpha=0.05$.

Results Behaviors were quantified based on Racine five-stage scoring and showed the significant epileptic generalized seizures in alive animal treated by intrastriatal injection of colchicine. However, tissue damage was invisible in the target brain area. Conclusion The colchicine, using injection successively into the dorsal striatal region of rat, can create recurring epileptic convulsions in the animal.
\end{abstract}

\section{Introduction}

Epilepsy, the chronic seizure convulsion, is known as the neural disorder with the rush activity of clusters of neurons that send out the wrong signals. Albeit, each type of the seizure is characterized by specific behavioral features, and researchers, so far, have tried to reproduce the abnormal neuronal discharges, as similarly in the animal models as seen in epileptic patients.

published online June 19, 2021
DOI https://doi.org/

$10.1055 / \mathrm{s}-0041-1727403$

ISSN 2213-6320
So far, the most frequently used models of chronic epilepsy and models of acute seizures have been induced by chemoconvulsants, traumatic brain injury, and electrical or sound stimuli. ${ }^{1}$ Even spontaneous recurrent seizures of rodents have recently been generated with chemoconvulsants. ${ }^{1}$ Considering this fact that every animal model has been played an important role in understanding of basic mechanisms underlying epilepsy, ${ }^{2}$ but one should not ignore that they have generally been used with expensive cost and

(C)2021. Indian Epilepsy Society.

Indian Epilepsy Society. This is an open access article published by Thieme under the terms of the Creative Commons Attribution-NonDerivative-NonCommercial-License, permitting copying and reproduction so long as the original work is given appropriate credit. Contents may not be used for commercial purposes, or adapted, remixed, transformed or built upon. (https://creativecommons.org/licenses/by-nc-nd/4.0/). Thieme Medical and Scientific Publishers Pvt. Ltd. A-12, 2nd Floor, Sector 2, Noida-201301 UP, India 
interfered with ethics. Kandratavicius et $\mathrm{al}^{1}$ documented that animal models of chronic epilepsy are not widely used because of time constraints and costs. Besides, for a given chemoconvulsant, one should rely on a specific group of neurons with detailed features aiming to study. Kainic acid, the L-glutamate analog, for the first time, was used to model epilepsy in rodents. ${ }^{3}$ Other compounds such as pentylenetetrazol (PTZ), strychnine, N-methyl-D, L-aspartate, tetanus toxin, and penicillin have been widely applied as acute seizure models, and not as animal models of epilepsy. ${ }^{4}$

Colchicine, an anti-inflammatory originally derived from the meadow saffron, Colchicum autumnale, can arrest mitosis in metaphase by interfering with the formation of spindle fibrils. Apart from inhibiting mitosis (a process heavily dependent on cytoskeletal changes), colchicine also checks neutrophil motility and activity, which leads to a strong anti-inflammatory effect. The alkaloid colchicine binds to the ends of microtubules and physically prevents the elongation of microtubule polymers. It can also depolymerize microtubule context and disturb axon transmission. ${ }^{5}$ It has also been previously indicated as a selective neurotoxin for dentate granule cells in the hippocampal formation of rats. ${ }^{6,7}$ This substance, furthermore, shares many other biological activities, such as binding to cell and nuclear membranes and inhibiting nucleoside transport and inflammatory responses. ${ }^{8}$

Some authors also adjusted the limbic seizures by colchicine in the rats, ${ }^{9,10}$ but, the animals hardly presented the motor activity typical of limbic seizures.

So far, a proper animal model has not been provided by the aid of colchicine that gives us the possibility that others may have chosen the inappropriate target place in the brain, or the innervations have mistakenly been involved. In fact, others did not introduce a suitable model for epilepsy with colchicine, and the problem seems to be due to one of three complications: the target and the dose and the route. ${ }^{1}$ Therefore, the present study is a kind of innovation in epilepsy modeling with the help of colchicine. We aimed to inject colchicine $(0.05-25 \mu \mathrm{g}$, intradorsal striatal of Wistar rat) to induce complementary motor cortical seizure, which differs with the kindled animal model in the hippocampus and amygdala.

\section{Materials and Methods}

\section{Animals}

Male Wistar rats (250-300 g) were used. They were housed in individual polyvinyl chloride cages at constant room temperature $\left(22 \pm 1^{\circ} \mathrm{C}\right)$ and maintained on a 12-hour light/dark cycle (lights on at 07:00 am). Food and water were available ad libitum. All experiments were performed under the guidelines for the care and use of laboratory animals of the Helsinki Declaration, and protocol was confirmed by the local animal ethics committee (Institutional documentation).

\section{Surgery}

For all surgical procedures, rats were anesthetized with ketamine (100 mg) -xylazine (20 mg). All stereotaxic coordinates for intracerebral manipulations were adapted from the atlas of Paxinos and Watson. ${ }^{11}$ The animals were implanted with bilateral stainless-steel guide cannulae (21-Gauge) at the dorsal striatum (AP: $0.5 \mathrm{~mm}$; L: $3 \mathrm{~mm}$; V: $3.6 \mathrm{~mm}$ ). These cannulae terminated $1 \mathrm{~mm}$ above the target areas. Stainless-steel stylets (od: $0.25 \mathrm{~mm}$ ) were inserted into guide cannulae to prevent their obstruction.

\section{Intrastriatal Injections}

Colchicine (sigma) was dissolved in $\mathrm{NaCl} 0.9 \%$. After a week's recovery, the rats were cannulated and received the substance $(0.05,0.5,1,3,5,9,15$, and $25 \mu \mathrm{g} / \mathrm{rat})$ intradorsal striatal by the aid of injection setup through 60 seconds. Injection cannulae (dental needle of 27-Gauge), which protruded one $\mathrm{mm}$ beyond the guide cannulae, were connected to a 5- $\mu$ Hamilton syringe by polyethylene tubing. At the end of the injection, the cannulae were left for a while, and then replaced by the stylets. Each animal received the drug for four consecutive days. The control group only received saline solution throughout the procedure. A delay of at least 24 hours was allowed between two injections.

\section{Behavioral and Pathological Assessments}

At the end of each injection, all the behavioral signs of experimental animals were recorded. The treated brain samples were collected and examined histopathologically (by the aid of hematoxylin-eosin staining). They were dissected out and fixed in $10 \%$ formalin. The dorsal striatal regions were cut coronally into 3 to- $4 \mu \mathrm{m}$-thick slices, embedded in paraffin and processed by the step section technique. The slices were eventually stained with hematoxylin-eosin and examined under the light microscope to verify the injection placement and possibility of lesions.

\section{Kindling by Colchicine}

Kindling was performed in the cannulated animal (at the dorsal striatum) a week after surgery. The recovered animal, from the 8th day, received intrastriatal injection of colchicine, for four consecutive days/once daily. After the injections, the animal's behavioral performance was recorded repeatedly and scored as follows:

Zero: no change,

One: contraction of the muscles of the mouth and face, and ears,

Two: axial contractions (shaking head or head-nodding),

Three: myoclonic contraction of the forelimbs and standing on two legs (rearing),

Four: general clonus seizures and falling on the side,

Five: falling on the back and general seizures (tonic-clonic).

To analyze the animals' behaviors, all behavioral aspects were repeatedly observed and recorded by video camera, and the records were reviewed for several times. The number of seizures per day and the length of time that each animal remained in phase five were also calculated.

\section{Statistics}

Neuronal density (number of neurons per unit area: $100 \mu \mathrm{m}$ ) was quantified at the target site and compared between the vehicle and experimental groups. Racine Scale scoring (from movement and contraction in the mouth and face to 
movement and shaking of the head to involuntary contractions of limbs and falling and standing) was measured and equated between the control and experimental groups. The difference of variances was shown by analysis of variance (ANOVA) under $\alpha=0.05$. The behaviors monitored by photo players were rechecked by an observer who was uninformed of the results, and the findings quantified based on 5-stage Racine scoring. ${ }^{12}$ These steps are mouth and face movement, head nodding, forelimb clonus, rearing (standing on hind legs) with forelimb clonus, and rearing and falling with forelimb clonus (generalized motor convulsions). These achievements were at last analyzed with the ANOVA.

\section{Results}

Epileptic Seizures in Animals Treated by Colchicine (the Percentages of Generalized Epilepsy)

Data obtained by monitoring of behavior in rats illustrate significant epileptic generalized seizures in animals treated by colchicine $(0.05-25 \mu \mathrm{g} / \mathrm{rat})$, intrastriatal, with no damaging effect in the target brain area (-Figs. 1-6). The behaviors quantified by Racine ${ }^{12} 5$-stage scoring showed the generalized motor convulsions, including rearing and falling with forelimb clonus.

Analysis by ANOVA and then Tukey's post hoc identified that the differences between the control and the experimental groups were statistically significant $(p<0.001)$.

\section{Duration of the Seizure (Stay in Stage 5) in the Experimental Rats}

Based on present data, by increasing the dose of colchicine, the duration of seizures (the delay time in stage five) increased, and behavioral symptoms became more pronounced.

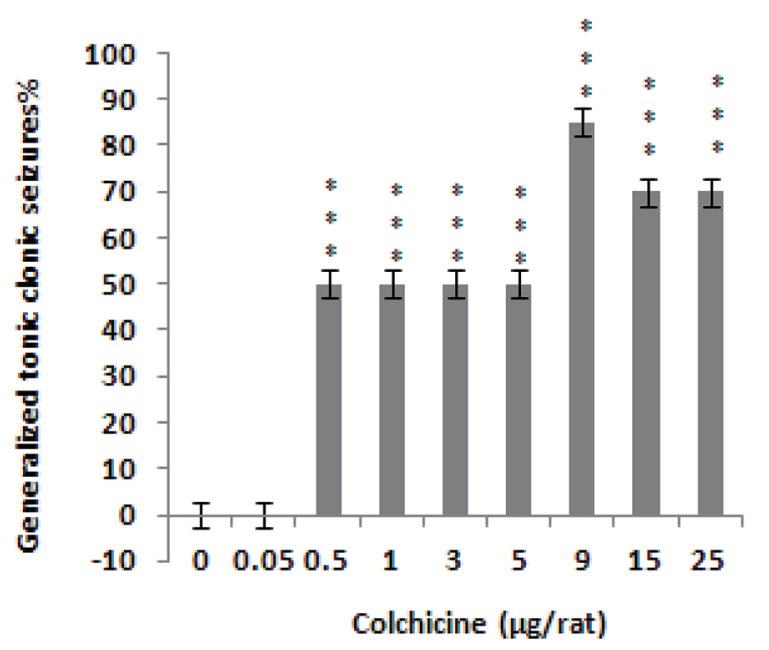

Fig. 1 Percentage of occurrence of generalized tonic-clonic seizures: Comparison of colchicine doses $(0.05-25 \mu \mathrm{g} /$ Wistar male rats, intrastriatal) on the percentage of generalized epilepsy attacks (Phase 5 seizures) with the control group (legend 0 ). Forty eight male rats received the colchicine during 4 consecutive days/daily once. A dose $(9 \mu \mathrm{g} / \mathrm{rat})$ most significantly showed the seizures. The data are presented according to analysis of variance and Tukey tests. $* * * p<0.001$.
The response was dose-dependent and significant $(p<0.001)$ (-Fig. 2), and the percentage of the duration of the seizure (stay in stage 5) was compared between the experimental rats, which were injected colchicine $(0.05,0.5,1,3,5,9,15$, and $25 \mu \mathrm{g} / \mathrm{rat}$ ) and the control, which were given intrastriatal injection of saline $0.9 \%$. The rats ( 6 per group) received the neurotoxin dose or saline for 4 consecutive days (daily once). According to the analysis, the differences between neurotoxin-treated and control groups were reasonably significant $(p<0.001)$.

\section{Comparison on the Time between Colchicine Injections and Specific Seizures}

The time between colchicine injections and specific seizures is shown in - Fig. 3. All animals (except the control group) received the identified doses of colchicine (intrastriatal, for four consecutive days/once daily). The analysis showed a significance result ( $p<0.05$ compared with control). According to the - Fig. 3 , the symptoms of generalized seizures were observed after injection of colchicine $0.5 \mu \mathrm{g} / \mathrm{rat}$. With increasing dose $(\sim 9 \mu \mathrm{g} / \mathrm{rat})$, the interval between seizure symptoms increased from the first injection of the alkaloid. However, with increasing doses (above the $9 \mu \mathrm{g} / \mathrm{rat}$ ), the time interval between the onset of symptoms and the time of colchicine injection were reduced by 1 day (the symptoms were seen after one injection). By viewing this figure and the number of seizures, it can be concluded that colchicine is approaching its toxic doses. (Between groups, differences based on Tukey's post hoc test are shown in the caption of the figure].

\section{Comparison on the Rate of Recurrence of Seizure in One Day (Due to Intrastriatal Injection of Colchicine with Different Doses)}

In - Fig. 4 , the rate of recurrence of seizure in 1 day (due to intrastriatal injection of colchicine) is shown. The injection (colchicine dose or saline) was performed according to the

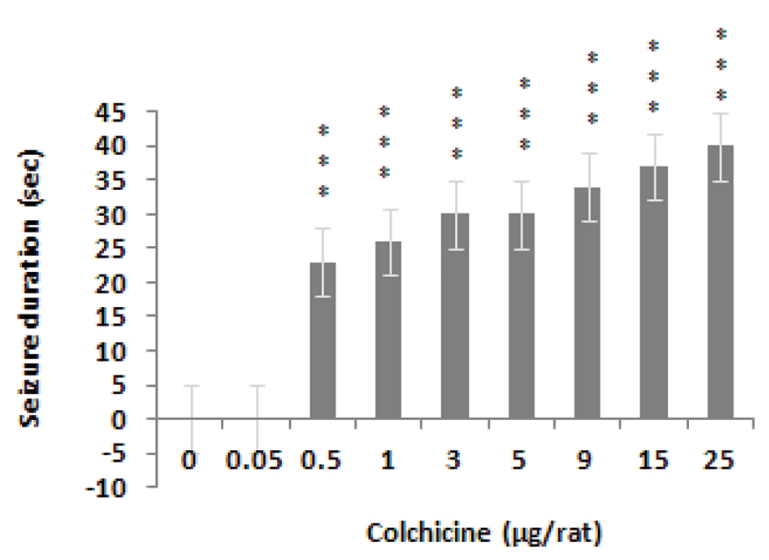

Fig. 2 The effect of intrastriatal injection of colchicine with specific doses on the duration of seizures: The figure compares the results of intrastriatal injection of colchicine doses $(0.05-25 \mu \mathrm{g} / \mathrm{rat})$ on the percentage of duration of seizure (stay in stage 5 ) with the control. A gradual increase in seizure duration with increasing dose of colchicine was observed. The data are presented according to the analysis of variance and Tukey tests: ${ }^{* *} p<0.001$. 


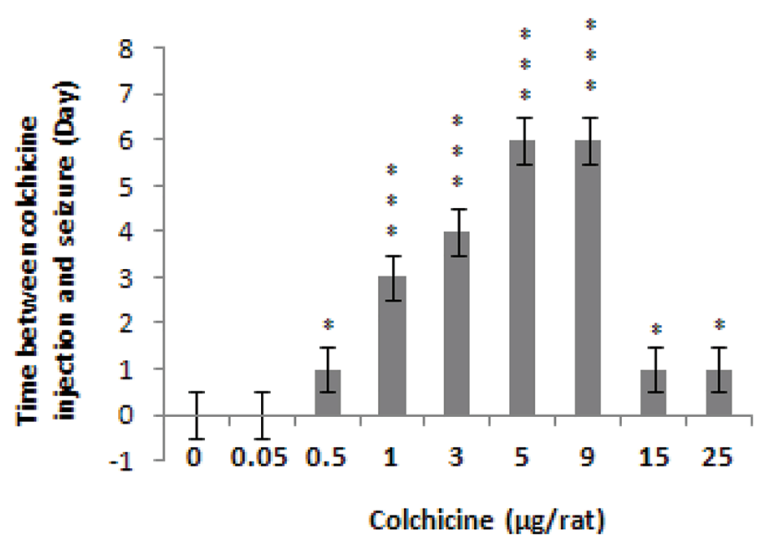

Fig. 3 Time between colchicine injection at specific doses and seizures: The figure compares the effects of intrastriatal injection of colchicine $(0.05-25 \mu \mathrm{g} / \mathrm{rat})$ on the time between colchicine injections and specific seizures, with the control group. The response was dose-dependent. Data are presented according to analysis of variance and Tukey test $\left({ }^{*} p<0.05,{ }^{* * *} p<0.001\right.$ compared with control).

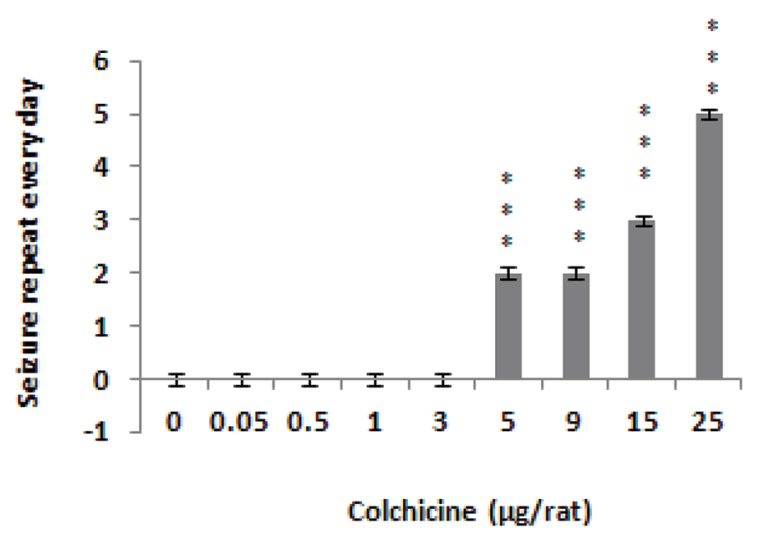

Fig. 4 The effect of intrastriatal injection of colchicine with specific doses on the repeat of seizures per day: This figure compares the effects of intrastriatal injection different doses of colchicine $(0.05$, $0.5,1,3,5,9,15$, and $25 \mu \mathrm{g} /$ rat) on the rate of recurrence of seizure in 1 day with control group. Recurrence was highest at the highest dose. The data are presented according to analysis of variance and Tukey test $\left({ }^{* * *} p<0.001\right.$ compared with control).

protocol (daily for 4 days). Based on achievements, the differences were significant $(p<0.001)$. By increasing the dose of colchicine, the number of seizures in 1 day increased. Recurrence of seizures did not observe in doses lower than dose $5 \mu \mathrm{g}$. Behavioral observations after injection of colchicine $25 \mu \mathrm{g}$ in rats showed recurrence of seizures at least five times a day (without animal death). By observing these results, the most effective dose for causing kindling with colchicine is $9 \mu \mathrm{g} /$ rat. By more injections of this dose $(9 \mu \mathrm{g})$, than the dose of $25 \mu \mathrm{g}$, the successive epileptic seizures occurred in animal, and the animal continued to live without being harmed. The desired response did not occur at lower doses (till $3 \mu \mathrm{g} / \mathrm{rat}$ ) proposing no irreversible changes in the neural organization at these doses.

\section{Percentage of Focal Epilepsy}

In - Fig. 5, the percentage of focal epilepsy is compared between the experimental rats and the control group. According to the findings, the differences were significant $(p<0.001)$. In focal epilepsy created in this animal model, only stage one (contraction of mouth and face, and ear muscles) and stage two (axial, head nodding) were observed. According to the figure, a dose $(9 \mu \mathrm{g})$, due to the high incidence of stage five seizures, has the lowest percentage of partial seizures. (In this dose, the generalized epilepsy [GE] occurs instead.)

\section{Tissue Histology in the Rats}

In - Fig. 6, a comparison of tissue image is shown between the rat that received colchicine and the control that was given saline into the dorsal striatum. As can be seen, the colchicine did not cause any neuronal degeneration in the target area. It should be noted that the criterion for showing the difference between tissue sections (control versus experimental) was to examine the density of neurons and neuronal projections in microscopic sections $(100 \mu \mathrm{m})$ using the image tool software and the results were used to describe the differences between them.

\section{Discussion}

Our aim was to introduce an animal model of epilepsy by injection of colchicine into the dorsal striatum of rat's brain. The results of monitoring the behavior of rats showed GE in the alive animals, which was dose-dependent. About 60\% of colchicine-treated rats showed GE, which occurred frequently; however, the neuronal destruction at the injection site was invisible compared with the control group.

A dose of the colchicine $(9 \mu \mathrm{g} / \mathrm{rat})$ had the highest fre-

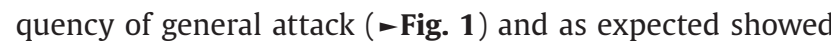
the lowest percentage of partial seizures ( - Fig. 5). At the

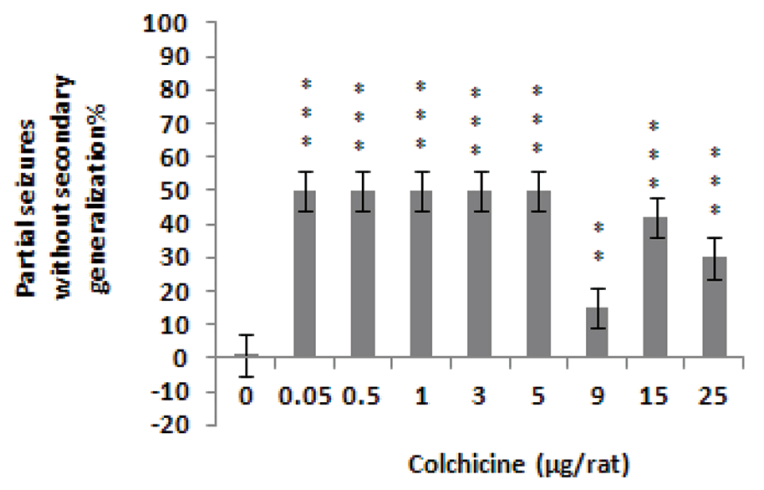

Fig. 5 Percentage of focal epilepsy: comparison of the effects of intrastriatal injection of colchicine $(0.05-25 \mu \mathrm{g} / \mathrm{rat})$ on the percentage of focal epileptic seizures (stage 2 seizures) with the control group. Partial seizures showed the lowest value at dose $9 \mu \mathrm{g} / \mathrm{rat}$, which had the most general attack. Data are presented according to the analysis of variance and Tukey tests $\left({ }^{* *} p<0.01,{ }^{* * *} p<\right.$ 0.001 compared with control). 

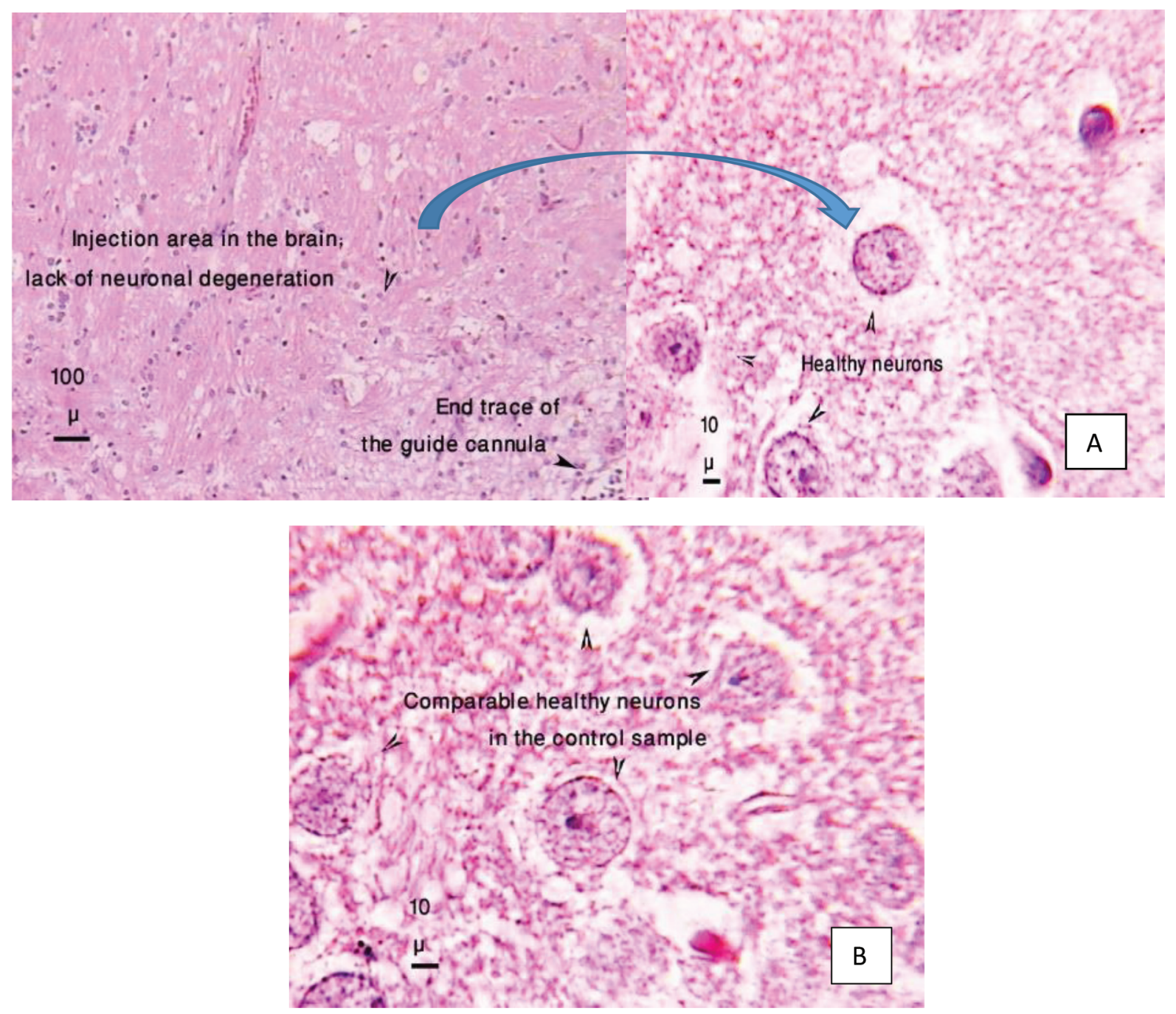

Fig. 6 Tissue evidence from colchicine injection site: this evidence was obtained by hematoxylin-eosin staining, and a comparison of tissue image between colchicine received rats $(\mathbf{A})$ and the control saline (B), intradorsal striatal, is shown in this figure. It can be seen that colchicine did not cause any neuronal degeneration in the target area. Line denotes the magnification.

lower doses, we observed only stage one (facial and ear muscle contraction) and stage two (head-nodding), and the onset of symptoms was delayed at the maximum dose ( $25 \mu \mathrm{g} / \mathrm{rat})$.

These results were obtained very fast by minimal invasive effect compared with kindling of the limbic areas. Because it has been indicated that daily, but prolonged electrical stimulation in some areas of the brain, such as the amygdala, leads to the gradual progression of limbic motor seizures. Short-term stimulation though causes changes in emotional behavior in animals. ${ }^{13}$

The duration of a seizure in the present model is very short (utmost 50 second) and close to human epilepsy.

Colchicine is a substance that disrupts axoplasmic transport and impairs neuronal function and disrupts neural processes. Despite the fact that animal models of epilepsy based on colchicine/its agonists such as thiocolchicoside have been previously proposed, the appropriate model of epilepsy was not introduced with these substances. Previously, an epileptogenic activity of both thiocolchicoside $\mathrm{e}^{14,15}$ and colchicine for gamma-aminobutyric acid (GABA) receptors has been suggested to explain the specific postsynaptic effects of the substances. Other findings, instead, have shown a certain reaction of this drug toward strychnine-binding sites in the rat brain, and in particular, strychnine-sensitive glycine receptors in the brain stem, spinal cord, and more rostral brain regions including the amygdala. ${ }^{16}$ An agonistic interaction of thiocolchicoside with spinal strychnine-sensitive glycine receptors likely mediate its myorelaxant activity. However, such interference can unlikely explain how it induces the motor seizure. Other researchers ${ }^{17}$ have also reported stable epileptiform discharges by intracellular studying of colchicine experimental epileptic focus.

We should consider that, so far, most animal models of epilepsy are kindled at the hippocampus and amygdala. Also, most types of live models that are tested for pathology and different efficacy of the drug are chemical seizures, kindling, and inflammation. Considering the documents, the dopaminergic neurotransmission within the striatum is 
involved in the control of GE seizures. ${ }^{18}$ More recently, both the caudate and the putamen nuclei (the dorsal striatum) of patients with ring chromosome 20 epilepsy have also been marked..$^{19}$ Others have additionally proposed the preventive effect of either GABA antagonist (bicuculline) or dopamine agonist (apomorphine) in a pilocarpine model in the rat striatum. They have suggested that not only dopamine but also GABAergic transmission in the striatum modulates the seizure propagation..$^{20,21}$ To remind, the importance of the caudate nucleus in the control of convulsive activity has already been found in the amygdaloid complex and the temporal cortex of the cat. ${ }^{22}$ The putamen cells have also been involved significantly during both frontal and motor seizures..$^{23}$ Besides, Vuong and Devergnas ${ }^{24}$ have recently indicated the role of basal ganglia in the control of motor cortical seizure development. Also, they have made electrolytic lesion of caudateputamen complex.

We injected colchicine for 4 days, consequentially/once per day, in the dorsal striatum of alive rat, a week after surgical procedure. After analyzing the behaviors of treated rats, in comparison to the control group, which solely received saline, we realized the colchicine treated animals as the epilepsy models. In fact, the epilepsy developed with colchicine in a dose-dependent manner. There was no attack at very low concentrations $(0.05 \mu \mathrm{g} / \mathrm{rat})$, and under more concentrations (0.5-5 $\mu \mathrm{g} / \mathrm{rat})$, the partial type occurred. In higher concentrations, rather than $5 \mu \mathrm{g} / \mathrm{rat}$, epilepsy was obtained and its duration showed increase at higher concentrations (between group analysis showed the differences at $\alpha=0.05$ ). This alkaloid seems to have an epileptogenic effect, which is a gradual process in which epilepsy develops in the normal brain, or changes in brain physiology lead to epilepsy. The amygdala though appears to have this property and plays a key role in epilepsy by disrupting glutamatergic and GABAergic synaptic transmission; ${ }^{25}$ however, according to this study, injection of effective doses of colchicine into the dorsal striatum over a short period (4 days) will cause such a complication. To discuss, colchicine as one of microtubule interfering agent inhibits microtubule assembly in the way of binding to $\beta$-tubulin, which results in curved tubulin dimer and prevents instant adoption between colchicine and tubulin subunits. ${ }^{26}$ As axonal transport depends almost entirely on microtubules, ${ }^{27}$ one may conclude that the neurotoxin interferes with axonal targeting signal. Thus, it may prevent distal transport in the axon to the cell soma and back, which causes imbalanced synaptic transmission leading to the error signal. The identification of such a signal that can power epileptic transport is unusual. It may encourage us to survey on the misinformation between the neurons alongside the dorsal striatum, which remain elusive.

We, furthermore, identified the brain structure of the treated rats, and compared with the control, which showed no significant changes ( - Fig. 6). In detail, striatal neurons can survive for extended periods with only insignificant troubles, while regular axonal transport could be predicted to break under these conditions if solely dependent on microtubule functions. Or else, there are several forms of axonal transport not associated with cytoskeleton motor elements that remained to be clarified. Alternatively, diffusion may be sufficient to maintain consistent levels of all neurotransmitters, including neuropeptides along the axon. In either case, an energetic mechanism of transport might become more relevant in situations of both slow and fast action potential firing.

Indeed, axonal transport is troubled in many neurodegenerative disorders such as ALS, Alzheimer's, Huntington's, and Parkinson's diseases. ${ }^{28}$ The neurotoxin colchicine, a substance that can bind to tubulin, and depolymerize microtubule, ${ }^{29}$ can encounter with the axonal trafficking. More recent approaches in the study of epilepsy are based on the use of a combination of optical and genetic methods. ${ }^{30}$ However, it should not be ignored that present criteria have low cost and proximity to general epilepsy. We know that the irreversible changes following excitability or changes in the structural organization of the neural network are involved in the development of kindling. The neuronal processes involved in kindling are related to the inability of the GABAaergic inhibitory system and increased N-methyl-D-aspartate receptor activity. Seizures are the result of an imbalance between excitatory and inhibitory processes, and research on the mechanisms of epilepsy focuses on changes in the activity of the major inhibitory (GABA) and excitatory amino acids (glutamate). ${ }^{31}$ However, other studies have shown that PTZ-induced kindling increases the destruction of hippocampal neurons and increases neurogenesis in dentate gyrus. ${ }^{32}$ Kainic acid that is a potent analog of glutamate and a potent agonist of glutamate receptor also is used to induce limbic seizures. Systemic or intraventricular use of this substance as others have shown leads to seizure activity that lasts for several hours, followed by a delayed phase in which no seizures are observed. However, swelling of neurons and perivascular astrocytes is observed following injection of this substance. For reasons that are not yet known, this substance even if injected systemically or even in an area away from the hippocampus can cause severe damage to the hippocampus. ${ }^{33}$ Neuronal death and decreased neuronal volume have also been reported with the use of other substances. ${ }^{34}$

The interesting results of the present study are that colchicine has no destructive effects on striatum neurons and no decrease in neuronal density was observed in the target area, while in other models resulting from chemical kindling, severe destruction of neurons in the target area has been observed. Behavior scoring was performed repeatedly during a day, as the colchicine injection was four times in 4 days not just once, without nerve damage, so, these results are not an acute transient effect. And this is the advantage of the colchicine model. The present results may suggest an idea and new animal model of epilepsy. In this model, the duration of a seizure is very short and close to human epilepsy, and is a maximum of 50 seconds. In the epileptic model of kindling with PTZ, it takes a few minutes, which bring the animal closer to death. But at the moment we do not have an explanation for our result and this requires further studies.

We reached these results by observing the behavioral signs along with studying the histology of the striatum and quantified the findings. However, the exact neuronal mechanisms remain to clarify. We plan to examine the local field potential 
in the dorsal striatal region of the model and record the brain waves frequency (study of power spectrum density).

\section{Conclusion}

In summary, we may conclude that the substance colchicine is a disturbance alkaloid of neural processes that can be used to create the recurrent alive epileptic model due to behavioral features with no lesion effect at the target area.

\section{Conflict of Interest}

None declared.

\section{Acknowledgment}

The authors thank the University for supporting of Graduate study.

\section{References}

1 Kandratavicius L, Balista PA, Lopes-Aguiar C, et al. Animal models of epilepsy: use and limitations. Neuropsychiatr Dis Treat 2014;10:1693-1705

2 Grone BP, Baraban SC. Animal models in epilepsy research: legacies and new directions. Nat Neurosci 2015;18(3):339-343

3 Sharma AK, Reams RY, Jordan WH, Miller MA, Thacker HL, Snyder PW. Mesial temporal lobe epilepsy: pathogenesis, induced rodent models and lesions. Toxicol Pathol 2007;35(7):984-999

4 White HS. Animal models of epileptogenesis. Neurology 2002; 59(9, Suppl 5) :S7-S14

5 Zaniani NR, Karami M, Porkhodadad S. Use of colchicine in cortical area 1 of the hippocampus impairs transmission of non-motivational information by the pyramidal cells. Basic Clin Neurosci 2013;4(4):323-328

6 Goldschmidt RB, Steward O. Preferential neurotoxicity of colchicine for granule cells of the dentate gyrus of the adult rat. Proc Natl Acad Sci U S A 1980;77(5):3047-3051

7 Daniels M. The role of microtubules in the growth and stabilization of nerve fibers. Ann N Y Acad Sci 1975;253:535-544

8 Porkhodadad S, Karami M, Jalali Nadoushan MR. Positive effect of nitric oxide on morphine-induced place conditioning in Wistar rats treated by colchicine intra-hippocampal CA1. J Clin Toxicol 2011;1:102

9 Oakley JC, Harneroff SR, Reynolds AF, Colchicine induced seizures: microtubules and epilepsy. Soc Neurosci Abs1981;630

10 Sutula T, Goldschmidt R, Steward O. Mechanisms of colchicine neurotoxicity in the dentate gyrus: dissociation of seizures and cell death. Exp Neurol 1983;81(3):683-693

11 Paxinos G, Watson C, The Rat Brain in Stereotaxic Coordinates. Orlando, FL: Academic Press; 2007

12 Racine RJ. Modification of seizure activity by electrical stimulation. II. Motor seizure. Electroencephalogr Clin Neurophysiol 1972;32(3):281-294

13 Kalynchuk LE. Long-term amygdala kindling in rats as a model for the study of interictal emotionality in temporal lobe epilepsy. Neurosci Biobehav Rev 2000;24(7):691-704

14 Biziere K, Huguet F, Narcisse G, Breteau M. Affinity of thiocolchicoside and thiocolchicoside analogues for the postsynaptic GABA receptor site. Eur J Pharmacol 1981;75(2-3):167-168
15 De Riu PL, Rosati G, Sotgiu S, Sechi G. Epileptic seizures after treatment with thiocolchicoside. Epilepsia 2001;42(8): 1084-1086

16 Cimino M, Marini P, Cattabeni F. Interaction of thiocolchicoside with $[3 \mathrm{H}]$ strychnine binding sites in rat spinal cord and brainstem. Eur J Pharmacol 1996;318(1):201-204

17 Reynolds AF, Oakley JC. The colchicine experimental epileptic focus: an intracellular study. Brain Res 1984;322(2):326-328

18 Deransart C, Lê B-T, Marescaux C, Depaulis A. Role of the subthalamo-nigral input in the control of amygdala-kindled seizures in the rat. Brain Res 1998;807(1-2):78-83

19 Biraben A, Semah F, Ribeiro M-J, Douaud G, Remy P, Depaulis A. PET evidence for a role of the basal ganglia in patients with ring chromosome 20 epilepsy. Neurology 2004;63(1):73-77

20 Turski L, Cavalheiro EA, Bortolotto ZA, Ikonomidou-Turski C, Kleinrok Z, Turski WA. Dopamine-sensitive anticonvulsant site in the rat striatum. J Neurosci 1988;8(11):4027-4037

21 Turski L, Diedrichs S, Klockgether T, et al. Paradoxical anticonvulsant activity of the aminobutyrate antagonist bicuculline methiodide in the rat striatum. Synapse 1991;7(1):14-20

22 La Grutta V, Amato G, Zagami MT. [The importance of the caudate nucleus in the control of convulsive activity in the amygdaloid complex and the temporal cortex of the cat]. Electroencephalogr Clin Neurophysiol 1971;31(1):57-69

23 Ono K, Mori K, Baba H, Wada JA. A role of the striatum in premotor cortical seizure development. Brain Res 1987;435(1-2): 84-90

24 Vuong J, Devergnas A. The role of the basal ganglia in the control of seizure. J Neural Transm (Vienna) 2018;125(3):531-545

25 Aroniadou-Anderjaska V, Fritsch B, Qashu F, Braga MF. Pathology and pathophysiology of the amygdala in epileptogenesis and epilepsy. Epilepsy Res 2008;78(2-3):102-116

26 Ravelli RB, Gigant B, Curmi PA, et al. Insight into tubulin regulation from a complex with colchicine and a stathmin-like domain. Nature 2004;428(6979):198-202

27 Maday S, Twelvetrees AE, Moughamian AJ, Holzbaur ELF. Axonal transport: cargo-specific mechanisms of motility and regulation. Neuron 2014;84(2):292-309

28 Millecamps S, Julien JP. Axonal transport deficits and neurodegenerative diseases. Nat Rev Neurosci 2013;14(3):161-176

29 Lu Y, Chen J, Xiao M, Li W, Miller DD. An overview of tubulin inhibitors that interact with the colchicine binding site. Pharm Res 2012;29(11):2943-2971

30 Cela E, Sjöström PJ. Novel optogenetic approaches in epilepsy research. Front Neurosci 2019. Doi:10.3389/fnins.2019.00947

31 Goudarzi E, Elahdadi Salmani M, Lashkarbolouki T, Goudarzi I. Hippocampal orexin receptors inactivation reduces PTZ induced seizures of male rats. Pharmacol Biochem Behav 2015;130:77-83

32 Yang J, Woodhall GL, Jones RS. Tonic facilitation of glutamate release by presynaptic NR2B-containing NMDA receptors is increased in the entorhinal cortex of chronically epileptic rats. J Neurosci 2006;26(2):406-410

33 Reddy DS, Kuruba R. Experimental models of status epilepticus and neuronal injury for evaluation of therapeutic interventions. Int J Mol Sci 2013;14(9):18284-18318

34 Akdogan I, Adiguzel E, Yilmaz I, Ozdemir MB, Sahiner M, Tufan AC. Penicillin-induced epilepsy model in rats: dose-dependant effect on hippocampal volume and neuron number. Brain Res Bull 2008;77(4):172-177 